\title{
The effect of sadness on global-local processing
}

\author{
Adrian von Mühlenen ${ }^{1} \cdot$ Lauren Bellaera ${ }^{2} \cdot$ Amrendra Singh $^{3} \cdot$ Narayanan Srinivasan $^{3}$
}

Published online: 4 May 2018

(C) The Psychonomic Society, Inc. 2018

\begin{abstract}
Gable and Harmon-Jones (Psychological Science, 21(2), 211-215, 2010) reported that sadness broadens attention in a globallocal letter task. This finding provided the key test for their motivational intensity account, which states that the level of spatial processing is not determined by emotional valence, but by motivational intensity. However, their finding is at odds with several other studies, showing no effect, or even a narrowing effect of sadness on attention. This paper reports two attempts to replicate the broadening effect of sadness on attention. Both experiments used a global-local letter task, but differed in terms of emotion induction: Experiment 1 used the same pictures as Gable and Harmon-Jones, taken from the IAPS dataset; Experiment 2 used a sad video underlaid with sad music. Results showed a sadness-specific global advantage in the error rates, but not in the reaction times. The same null results were also found in a South-Asian sample in both experiments, showing that effects on global/local processing were not influenced by a culturally related processing bias.
\end{abstract}

Keywords Sadness $\cdot$ Global-local processing $\cdot$ Emotion induction

\section{Introduction}

People often refer to "seeing red" as a way of conveying the fact that they cannot attend to anything but the source of their anger and to "feeling blue or down" as a way of expressing a person's preoccupation with their own problems. Such high levels of focused attention when experiencing negative emotional states is not only associated with the trigger or source of the emotion but can also affect how the general surrounding is perceived. One study going back over 50 years suggested that negative emotions focus attention (Easterbrook, 1959). This finding has been confirmed in more recent studies, and it also has been shown that positive emotions broaden attention (e.g., Fredrickson \& Branigan, 2005; Gasper \& Clore 2002; Rowe, Hirsch, \& Anderson, 2007; Uddenberg \& Shim, 2015). In particular, emotional states are used as a way to guide the level

Adrian von Mühlenen

A.vonmuhlenen@warwick.ac.uk

1 Department of Psychology, University of Warwick, Coventry CV4 7AL, UK

2 Faculty of Education, University of Cambridge, Cambridge, UK

3 Centre of Behavioural and Cognitive Sciences, University of Allahabad, Allahabad, India of processing most appropriate in a given context. For instance, the experience of negative emotions indicates that something is wrong and thus a focused approach is needed to solve the problem. In contrast, positive emotions suggest that everything is okay and consequently a less focused approach in which a person can expand their cognitions is appropriate. This valence-based distinction is the foundation for several affect-related theories (e.g., Clore, Wyer, et al., 2001; Derryberry \& Tucker, 1994) and more generally the understanding between affect and cognition is often explained in terms of negative and positive valence.

An important question is whether this distinction applies to all emotions in the same way. Gable and Harmon-Jones $(2008,2010)$ have recently addressed this question and they suggest that the level of spatial processing is not determined by emotional valence, but by motivational intensity, that is the urge to act, which is thought to be similar to but not the same as arousal (Bradley \& Lang, 2007; Gable \& HarmonJones, 2013). The difference being that arousal is non-specific, whereas motivational intensity is closely associated with goal-directed behavior, which primarily determines the level of attentional focus (Gable \& Harmon-Jones, 2010). Emotions high in motivational intensity precede goal-directed behavior and thus attention is narrowed in order to facilitate goals, whereas emotions low in motivational intensity follow the attainment (or failure) of such goals and thus attention is broadened in order to expand 
cognitive processes after a goal has passed. For example, sadness is associated with a failure to obtain goals, which should therefore broaden attention to aid the consideration of alternative options.

In support of their theory, Gable and Harmon-Jones (2010) reported a study showing that sad images selected from the International Affective Picture System (IAPS) database (Lang, Bradley, \& Cuthbert, 2005) broadened attention in a global-local letter task (Navon, 1977). That is, reaction times (RTs) to the global letter were faster when preceded by a sad image compared to when preceded by a neutral image. Similarly, they reported that disgust, a negative emotion high in motivational intensity, narrowed attention (Gable \& Harmon-Jones, 2010). They also reported the same pattern for positive emotions: humor, low in motivational intensity broadened attention, whereas desire (i.e., films showing delicious deserts), high in motivational intensity narrowed attention (Gable \& Harmon-Jones, 2008; for a recent replication of this study, see Domachowska et al., 2016; Gable \& HarmonJones, 2016). Based on these studies they concluded that motivational intensity - and not valence per se - accounted for the differences in global-local processing.

Gable and Harmon-Jones' (2010) finding that sadness has a broadening effect on attention is pivotal for their motivational framework because sadness is the only negative emotion with a low motivational intensity. Yet this finding seems to be at odds with a number of other studies that either showed sadness had no effect on attention, or even the opposite - a narrowing effect on attention. One of the earlier studies to demonstrate such a narrowing effect was by Gasper and Clore (2002) who found that judgments in a shape task (i.e., Kimchi \& Palmer, 1982) were made more at a local level if participants were asked to recall sad memories, and more at a global level when they were asked to recall happy memories. More sustained forms of sadness have also shown this pattern of results, with depressed individuals displaying an inverse relationship to global processing; that is, depressed people were less likely to process at the global level (Basso, Schefft, \& Dember, 1996). Further, Melcher, Obst, Mann, Paulus, and Gruber (2012) revealed that even briefly displayed affective cues, such as sad-related primes, reduced RT in incongruent trials at a stimulus-based level (e.g., Stroop task) and at a space-based level (e.g., flanker task). Table 1 presents a non-exhaustive list of empirical studies looking at the effect of "sadness" on attentional breadth, separately for narrowing effect, no effect, or a broadening effect. In one of our own studies we used a modified flanker task and found less spatial interference after participants were watching a sad video compared to a neutral video (Bellaera \& von Mühlenen, 2017).

The idea that sadness narrows attention fits within a wider framework of affect-cognition studies which show that sadness is closely associated with an analytic, bottom-up, and detail-orientated processing style (see Clore \& Palmer, 2009 for a review). A functional reason for this might be that a sad emotional state indicates that a problem needs to be solved, which requires a thorough analysis of the situation, or, alternatively, a sad emotional state might help distract a person from the cause of their sadness (Bodenhausen, Sheppard, \& Kramer, 1994).

Recent findings have shown that the relationship between cognition and emotion is bidirectional; that is, perceptual processing breadth also influences the processing of emotion (Duncan \& Barrett, 2007). Based on the work discussed above it would be anticipated that a global focus would facilitate the processing of positive emotions, whereas a local focus would enhance the processing of negative emotions. Recent work by Srinivasan and colleagues showed that this is indeed the case, with the identification and recognition of happy faces being enhanced under conditions of broad attention and the identification and recognition of sad faces being enhanced during focused attention (Srinivasan \& Gupta, 2010; Srinivasan \& Hanif, 2010). This bi-directional relationship of global-happy and local-sad further supports the notion that sadness and analytic processing are strongly linked (Clore \& Palmer, 2009).

Alternatively, the relationship between emotion and attention may not be fixed; the flexibility hypothesis (Huntsinger, Clore, \& Bar-Anan, 2010) proposes that positive emotions and negative emotions are not always associated with global processing and local processing. Instead, emotional states convey value about the response orientations, which are interpreted as either "go" signals or "stop" signals. For example, when a person is in a positive emotional state they tend to accept the current attentional state (i.e., a "go" signal), whereas someone in a negative emotional state rejects it (i.e., a "stop" signal) (Huntsinger, 2012; Huntsinger, Clore, \& BarAnan, 2010). Thus, if a local focus is dominant in a given context then a positive mood state will further facilitate this attentional state. In contrast, a negative emotional state will lead to a global focus being adopted as the more accessible "narrow" attentional scope is rejected. In support of this, Tan, Jones and Watson (2009) showed that positive affect led to faster responses to local targets for participants who were by default more globally biased.

It should also be noted that four of the studies listed in Table 1 revealed neither a narrowing nor a broadening effect of sadness (i.e., Finucane et al., 2010; Fredrickson \& Branigan, 2005; Rowe et al., 2007; Uddenberg \& Shim, 2015). One possible reason for these null findings might also be the difficulty in inducing sadness in an experimental setting (e.g., Rowe et al., 2007), especially without inducing related feelings, such as guilt, shame, anger, or fear (e.g., Kringelbach \& Phillips, 2014). This could be one reason why, in comparison to fear, there is relatively little published research on the effect of induced sadness on cognition (see Chepenik, Cornew, \& Farah, 2007). Moreover, because the existing studies used different procedures for emotion induction 
Table 1 A summary of empirical studies, which explicitly investigated the relation between sadness and spatial attentional breadth

\begin{tabular}{lll}
\hline Narrowing effect & No effect & Broadening effect \\
\hline Basso et al. (1996) & Finucane, Whiteman, \& Power (2010) & Gable \& Harmon-Jones (2010) \\
Fenske \& Eastwood (2003) & Fredrickson \& Branigan (2005) & \\
Gasper \& Clore (2002) & Rowe, Hirsch, \& Anderson (2007) & \\
Melcher et al. (2012) & Uddenberg \& Shim (2015) & \\
\hline
\end{tabular}

and different attention measures, it is difficult to establish the source of any discrepancies in findings.

The present study aims to investigate whether sadness narrows or broadens attention. It uses exactly the same stimuli and procedure as Gable and Harmon-Jones (2010) and tests two different groups of participants, one sample from the UK (Experiment 1a) and another sample from India (Experiment 1b). The primary purpose for this replication is to provide more evidence for Gable and Harmon-Jones motivational intensity account. Moreover, this replication includes a South-Asian sample, in addition to a Western sample, in order to add a crosscultural element to the study. This is important because previous studies have shown that individuals growing up in a South-Asian culture may have a more holistic perceptual style and a more global attentional focus than individuals growing up in a Western culture (e.g., Boduroglu, Shah, \& Nisbett, 2009; Masuda \& Nisbett, 2001). Hence, this will allow us to assess in what way such cultural related processing biases modulate the effect of sadness on attention. If the Indian sample shows a more global focus than the UK sample, then according to Gable and Harmon-Jones's account, sadness should produce an even larger focusing effect in the Indian sample (e.g., because there is more room for such an effect)

\section{Experiment 1}

Experiment 1 was aimed at replicating Gable and HarmonJones' (2010) first experiment with sad and neutral pictures, with a UK sample (Experiment 1a) and an Indian sample (Experiment 1b).

\section{Method}

Participants We aimed to recruit 40 participants from the University of Warwick research participant pool and 40 participants from the University of Allahabad participant pool as volunteers to take part in the study. ${ }^{1}$ Participants reported normal or corrected-to-normal vision and were not aware of the

\footnotetext{
${ }^{1}$ The number of participants was based on a power analysis using Faul, Erdfelder, Lang, and Buchner's (2007) G*Power software (achieving a power of 0.95), where Cohen's (1988) d was estimated from the results of a previous study by Bellaera, von Mühlenen, and Watson (2014) that used a global-local task. Note that this number is similar to Gable and Harmon-Jones' (2010) who tested 35 participants.
}

purpose of the study. Each participant gave informed consent and the study was approved by the Ethics Committees of University of Warwick and University of Allahabad.

Apparatus and stimuli Each participant completed the experiment individually. The experiment took place in a dimly lit sound-attenuated room using a 19-in. LCD monitor $(60 \mathrm{~Hz}$, $1,440 \times 900$ resolution). Each participant sat with their head approximately $57 \mathrm{~cm}$ away from the computer screen. The left and right arrow keys of a computer keyboard were used to record responses. Custom-written software was used to generate stimuli and record responses.

Thirty-two pictures from the IAPS database ${ }^{2}$ were used (Lang, Bradley, \& Cuthbert 2005), 16 of which were unpleasant and low in arousal (i.e., sadness) and the rest were neutral. Each picture subtended $26^{\circ} \times 20^{\circ}$ in size and was presented on a black background at the center of the screen. The sad pictures and the neutral pictures were matched for color, brightness, object size, and human presence. The Self-Assessment Manikin (Bradley \& Lang, 1994) was used to rate each picture in terms of pleasure and arousal at the end of the experiment.

The Navon letter task was used to measure attentional breadth. In this task, the stimuli consisted of a larger letter (width $1.91^{\circ}$ and height $3.34^{\circ}$ ) made up of nine smaller letters (width $0.32^{\circ}$ and height $0.48^{\circ}$, line thickness 1 pixel). The stimulus always contained the letter " $\mathrm{H}$ " or " $\mathrm{T}$ " (target letters) and the letter "L" or "F" (distractor letters). For example, a large T consisting of smaller $L$ s (see Fig. 1). The stimuli were gray (RGB values: $128,128,128$ ) presented on a black background. Stimuli included a fixation cross (size $0.6^{\circ}$ visual angle).

Design The main independent factors included Picture Type (sad, neutral) and Target Type (global, local). The dependent variables were RT and error rate. There were in total 32 experimental trials (16 local target trials, 16 global target trials). Experiment 1a used a sample in the UK (University of Warwick) whereas Experiment $1 \mathrm{~b}$ used a sample from India (University of Allahabad).

\footnotetext{
${ }^{2}$ Negative IAPS numbers: 2205, 2455, 2490, 2590, 2700, 2795, 3300, 9000 9001, 9190, 9220, 9331, 9341, 9390, 9471, 9912 Neutral IAPS numbers: 2102, 2191, 2393, 2500, 5535, 5740, 7057, 7205. In addition, we used eight neutral pictures that were selected by Gable and Harmon-Jones (2010) from the Internet - which they kindly made available to us on request.
} 

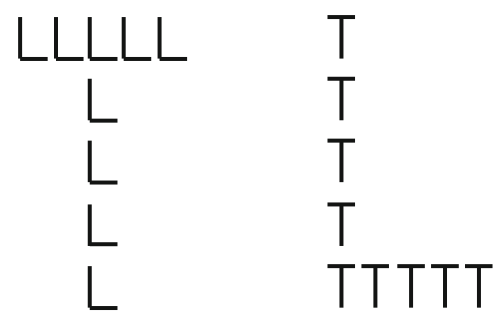

\section{(a) Global level}

(b) Local level

Fig. 1 Examples of compound stimuli used in Experiment 1. The target letter " $T$ " is presented either at (a) the global level, or at (b) the local level

Procedure On arrival, participants filled in a consent form and read the written instructions. They then completed a block of practice trials, which used neutral picture stimuli.

A trial began with a central fixation cross, presented for $3 \mathrm{~s}$ followed by an IAPS picture presented for $6 \mathrm{~s}$. This was followed by another fixation cross, displayed for $500 \mathrm{~ms}$ and then a Navon letter. Participants were instructed to report which target letter was present (H or T). They pressed the left arrow key if the $\mathrm{T}$ was present and the right arrow key if the $\mathrm{H}$ was present. Participants were asked to respond as quickly and accurately as possible, making less than $5 \%$ errors overall. The Navon letter was presented on the screen until a response was made or $5 \mathrm{~s}$ had passed; between each trial there was 8- to 10 -s interval. Participants completed six neutral practice trials in order to familiarize themselves with the task before starting the experimental blocks.

After the Navon letter task was completed, the same pictures were shown again for $2 \mathrm{~s}$ and participants had to rate them in terms of pleasure and arousal using the self-assessment manikin (Bradley \& Lang, 1994). In addition, participants also rated how sad each image made them feel $(1=$ no sadness, $9=$ strongest sadness). Finally, the Differential Emotions Scale (DES, Izard, Dougherty, Bloxom, \& Kotsch, 1974; Philippot, 1993), which consists of ten emotion categories (interest, amusement, sadness, anger, fear, anxiety, disgust, contempt, surprise, and elatedness), was used to assess the participants' discrete emotional states. Participants had to rate on a five-point scale from one ("not at all") to five ("very strongly") the extent to which these adjectives corresponded to their current emotional state.

Analysis Mean percentage errors were calculated for each participant and variable combination. Likewise, mean RTs were calculated excluding errors and RT outliers $(>3 S D$ from cell mean). ${ }^{3}$ Both individual mean errors and individual mean RTs

\footnotetext{
${ }^{3}$ Gable and Harmon-Jones (2010) used a log transformation on the RTs. They did not provide an explanation for this transformation. We have therefore analyzed the data with and without the log transformation, but we report here only the non-transformed RTs, as the outcome of the two analyses did not differ.
}

were analyzed with a three-way mixed design Analysis of Variance (ANOVA) with the within-subject factors Target Type (local, global) and Picture Type (sad, neutral) and the between-subject factor Sample (UK, India). Significant interactions were further analyzed by separate ANOVAs and ttests.

\section{Results}

In total 46 participants signed up in the UK and 40 participants in India to take part in the experiment. Six participants from the UK sample were not included in the analysis because they declared to be of Asian origin (China 3; Malaysia 2; Sri Lanka 1). ${ }^{4}$ Furthermore, four participants from the India sample had to be excluded because a post-experimental analysis revealed that they did not correctly follow task instructions: They wrongly responded only to the identity of the global letter that led to $100 \%$ errors in conditions where the target was a specific local letter. Thus, the following results include 40 participants from the UK and 36 participants from the India sample.

Navon letter task Mean RTs (excluding 2.8\% errors and $0.4 \%$ outliers) were calculated separately for the UK and the India sample (see Fig. 2). The three-way ANOVA revealed a significant main effect for Sample, $F(1,74)=5.08, p=.03$, and for Target Type, $\mathrm{F}(1,74)=5.22, \mathrm{p}=.03$ : RTs were overall $165 \mathrm{~ms}$ faster in in the UK than in the India sample; and RTs were overall $42 \mathrm{~ms}$ faster for global than for local targets. Figure 2 further suggests that the global advantage was overall less pronounced in the UK sample than in the India sample (cf. left and right graph); however, the 2-way interaction was not statistically significant, $F(1,74)=1.11, p=.29$. Note that none of the effects involving Picture Type was significant (e.g., Target Type $\times$ Picture Type, $\mathrm{F}(1,74)=0.08 \mathrm{p}=.78$; 3-way interaction, $\mathrm{F}(1,74)=0.05, \mathrm{p}=.82)$. The absence of the Target Type $\times$ Picture Type interaction was further substantiated with a Bayesian ANOVA using the JASP software (Version 0.8.0.0; JASP Team, 2016), which showed strong evidence in favor of the null hypothesis $\left(\mathrm{BF}_{\text {Inclusion }}=0.06\right.$; see Wagenmakers, 2007; Wetzels et al., 2011). The pattern of results was very similar with logarithmically transformed RTs $($ Target Type $\times$ Picture Type, $\mathrm{F}(1,74)=0.08 \mathrm{p}=.78$; 3way interaction, $F(1,74)=0.15, p=.68)$.

The overall error rate was relatively low $(2.8 \%)$, confirming that the participants who were included in the analysis had no trouble following the task instruction of keeping errors below $5 \%$. This rate is also comparable to the $2.9 \%$ reported by Gable and Harmon-Jones (2010). The three-way ANOVA revealed a significant interaction between Target

\footnotetext{
${ }^{4}$ Selective recruitment based on origin was not possible; the excluded participants were therefore successively replaced with additional ones.
} 

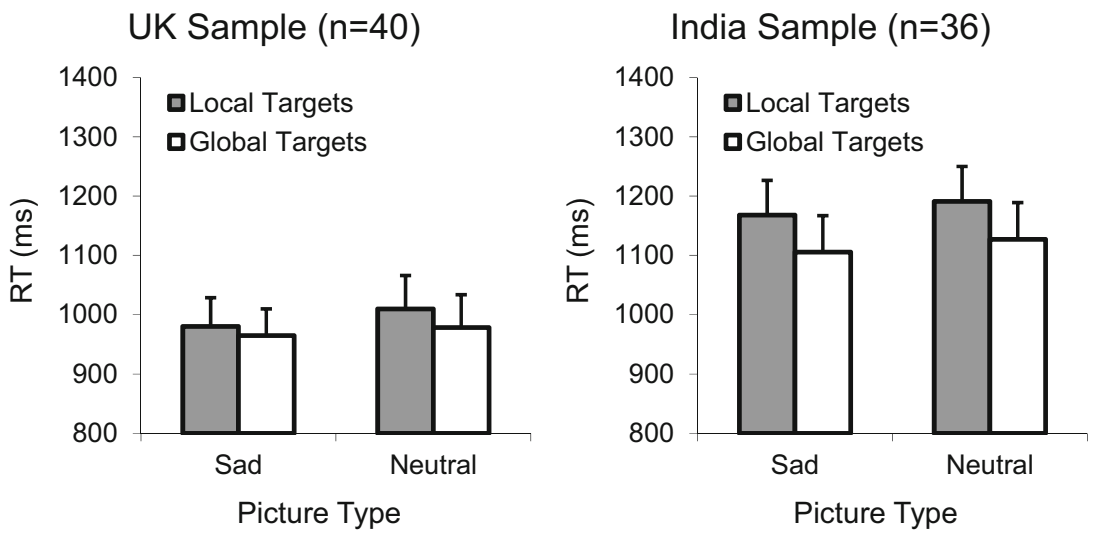

Fig. 2 Mean reaction times (RTs) and SEM in Experiment 1 as a function of picture type (sad or neutral) and target type (local or global), in separate graphs for the UK sample and the India sample

Type and Picture Type, $\mathrm{F}(1,74)=10.85, \mathrm{p}=.002$ (note that the same interaction was also significant when errors were $\log$ transformed). This interaction is illustrated in Fig. 3 (left graph), which shows the pattern consistent with Gable and Harmon-Jones study, with a global advantage following sad pictures, but not neutral pictures. It is hence possible that the RT results reported above are distorted by speed-accuracy trade-offs (i.e., faster responses at the cost of more errors). In order to explore this possibility, RTs were adjusted for errors by calculating inverse efficiency score, where RTs are divided by accuracy (see Townsend and Ashby, 1983). The Target Type by Picture Type effects with inverse efficiency score is illustrated in Fig. 3 (right graph). Even though the graph shows numerically a somewhat larger global advantage following sad pictures, the respective two-way interaction was statistically not significant, $\mathrm{F}(1,74)=1.24, \mathrm{p}=.27$, and neither was the three-way interaction, $\mathrm{F}(1,74)=0.18, \mathrm{p}=.67$.

IAPS picture ratings Participants rated each picture on a ninepoint scale in terms of pleasure, arousal, and sadness. Participants in the UK sample tended to give lower ratings than participants in the India sample (3.5 vs. 4.3, respectively), possibly due to cultural differences in response style (e.g., Mottus et al., 2012). Table 2 reports the average picture ratings conjoined across both samples. As can be seen, the picture manipulation worked very well: Sad pictures were rated as less pleasurable, $\mathrm{t}(74)=20.36, \mathrm{p}<.001$, and as sadder, $\mathrm{t}(74)$ $=28.04, \mathrm{p}<.001$, than neutral pictures.

In a further exploration, the sadness rating of each picture was plotted against the corresponding RT of the Navon letter task, using different symbols for picture types and target types. The resulting graph (see Fig. 4) shows that picture types nicely split into two distinct sadness categories (neutral and sad) with the exception of one picture in the sad category, which had an atypical low sadness score (IAPS 9220, showing a kitchen sink with dirty dishes). Removing trials using this particular picture did not change the overall pattern of result in the Navon letter task. The graph also shows no indication of a unique global advantage following sad picture, that is, responses to global targets (white symbols) were not particularly fast following sad pictures (triangles) when compared with the respective pattern of responses following neutral pictures (circles).
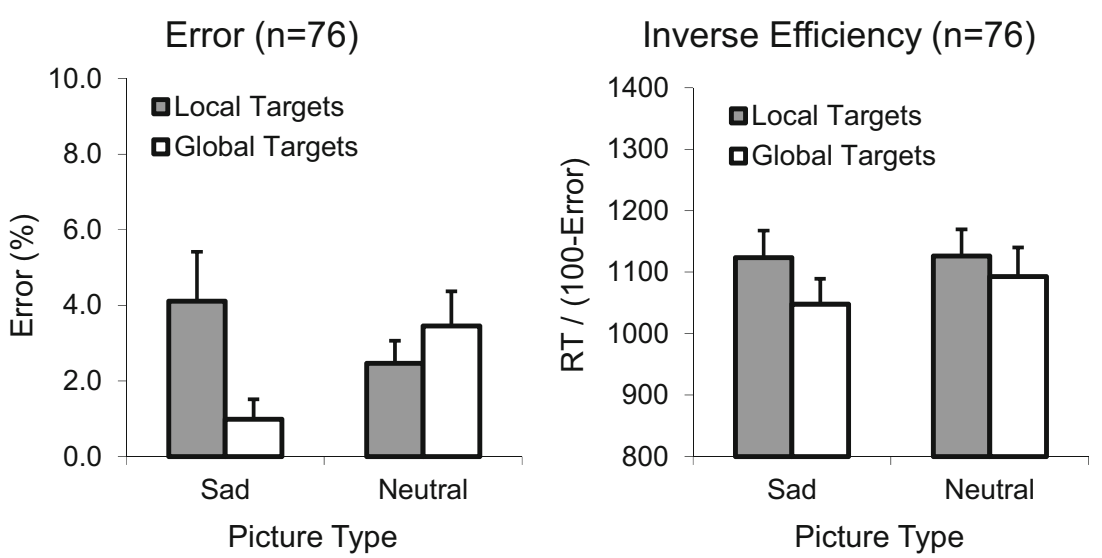

Fig. 3 Mean errors (left graph) and mean inverse efficiency scores (right graph) in Experiment 1 (averaged across both samples) as a function of picture type and target type 
Table 2 Pleasure, arousal, and sadness ratings averaged across participants from both samples (UK and India) in Experiments 1, separately for sad and neutral pictures

\begin{tabular}{lccc}
\hline & \multicolumn{3}{c}{ Rating } \\
\hline Picture type & Pleasure & Arousal & Sadness \\
Sad & 3.3 & 3.4 & 5.8 \\
Neutral & 6.0 & 3.1 & 2.0 \\
\hline
\end{tabular}

Differential emotions scale (DES) The emotional ratings collected at the end of the experiment were lower in the UK sample than in the Indian sample (1.9 vs. 2.5 , respectively), with the exception of sadness (2.5 vs. 2.2 , respectively). Again, we can only speculate that this might have to do with cultural variations in the response style (e.g., Mottus et al., 2012). In the following analysis, the ratings were combined across both samples (see first column in Table 3). Next, we used the sadness rating to predict the attentional focus in the Navon task. First, we calculated for each participant two global advantage scores - one for each picture type - by subtracting global RTs from local RTs. Subsequent correlation analysis showed no link between participants' sadness rating and the average global advantage score (i.e., averaged across both picture types), $r=.02, p=0.87$. This suggests that the attentional focus in the Navon task did not depend on participants' self-rated sadness. There was also no link between sadness and the relative focus score (i.e., the difference score between both picture types), $r=-.04, p=.73$. This further suggests that the possible effect of sad pictures on attention did not depend on the participants' self-rated sadness. Moreover, neither of these two scores (global advantage or focus) was linked with any of the other nine emotion categories (all $\mathrm{r}$ between -.09 and .13), and it was the same when scores were based on inverse efficiency scores (all $r$ between -.11 and .17) rather than on RTs.

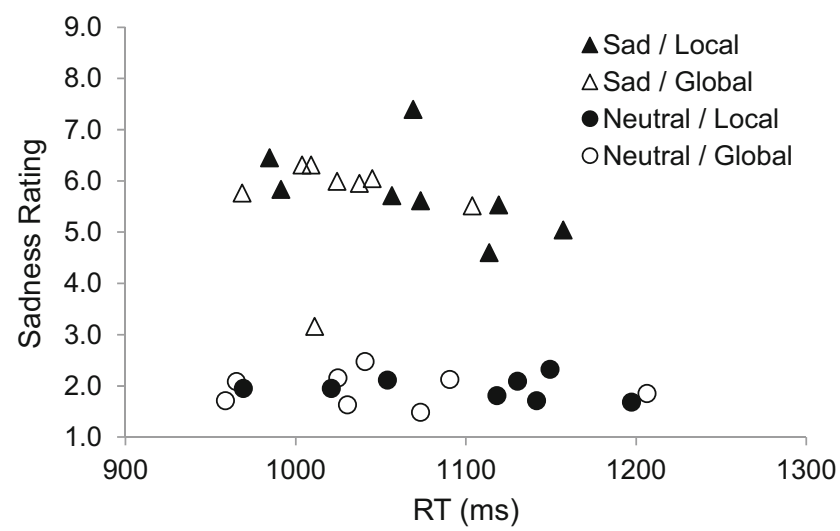

Fig. 4 Sadness rating for each picture plotted as a function of RT in the Navon letter task, with different symbols for picture type (neutral, sad) and target type (local, global)

\section{Discussion}

The primary purpose of Experiment 1 was to replicate Gable and Harmon-Jones (2010) study using the same stimuli and procedure. According to their motivational intensity account, it was predicted that sad pictures would have a broadening effect on the attentional focus, facilitating the response to global targets compared to local targets. This prediction was not supported by the current findings, at least not when looking at the RTs. However, a sadness-specific global advantage occurred in the error rates. Note that the overall error rate was in the same range as in Gable and Harmon-Jones' study ( 2.8 vs. $2.9 \%$, respectively). However, Gable and HarmonJones concluded that there were insufficient errors to permit analyses of error rates, so we can only speculate whether their data would have shown the same effect or not. We would like to point out that the error rate was relatively small and based on only eight trials. Moreover, when we adjusted the RTs for errors by the inverse efficiency calculation, the overall pattern of results did not substantially differ from the original pattern of RT results.

The RTs showed an overall global advantage, in line with Navon's (1977) notion that global processing takes precedence over local. However, in contrast to Gable and Harmon-Jones (2010), this effect was not more pronounced with sad pictures than with neutral pictures $(28$ vs. $47 \mathrm{~ms}$, respectively). A detailed analysis including picture ratings and mood self-ratings showed that the absence of an RT effect is not due to a decreased emotionality of the sad pictures or an unrelated effect of participants' mood. The overall difference between sad and neutral pictures was in fact higher in the current study than in Gable and Harmon-Jones' study, in terms of both sadness rating (3.8 vs. 2.6, for our and their study, respectively) and pleasure rating ( 2.7 vs. 2.0 , respectively).

Table 3 The mean subjective ratings for each emotion category in Experiment 1 and in Experiment 2

\begin{tabular}{|c|c|c|c|c|c|}
\hline \multirow[b]{3}{*}{ Emotion } & \multirow{3}{*}{$\begin{array}{l}\text { Expt. } 1 \\
\text { IAPS } \\
\text { T2 }\end{array}$} & \multicolumn{4}{|c|}{ Expt. 2} \\
\hline & & \multicolumn{2}{|c|}{ Sad video } & \multicolumn{2}{|c|}{ Neutral videc } \\
\hline & & $\mathrm{T} 1$ & $\mathrm{~T} 2$ & $\mathrm{~T} 1$ & $\mathrm{~T} 2$ \\
\hline Interested, concentrated, alert & 3.5 & 3.8 & 3.5 & 3.1 & 3.4 \\
\hline Amused, Joyful, merry & 2.8 & 1.4 & 2.2 & 2.6 & 2.5 \\
\hline Sad, down-hearted, blue & 2.3 & 3.6 & 2.7 & 1.7 & 2.7 \\
\hline Angry, irritated, mad & 1.6 & 2.2 & 1.8 & 1.4 & 1.7 \\
\hline Fearful, scared, afraid & 1.6 & 2.8 & 1.8 & 1.3 & 1.5 \\
\hline Anxious, tense, nervous & 2.2 & 2.9 & 2.0 & 1.9 & 2.0 \\
\hline Disgusted, turned-off, repulsed & 1.8 & 2.8 & 1.9 & 1.6 & 1.7 \\
\hline Disdainful, scornful, contemptuous & 1.7 & 2.1 & 1.8 & 1.7 & 1.8 \\
\hline Surprised, amazed, astonished & 2.1 & 2.1 & 2.0 & 1.6 & 2.2 \\
\hline Warm-hearted, gleeful, elated & 2.6 & 1.4 & 2.2 & 2.3 & 2.4 \\
\hline
\end{tabular}


The absence of an effect can also not be explained with an ambiguous division of pictures into sadness categories. The sadness ratings of each picture (see Fig. 4) showed (with one exception) a very clear-cut separation between sad and neutral pictures. Finally, RT results were also not influenced by the self-rated sadness, which was collected at the end of the experiment.

Another purpose of Experiment 1 was to include a crosscultural factor by running two samples, one from a Western culture and one from a South-Asian culture. Based on previous studies (Boduroglu, et al., 2009; Masuda \& Nisbett, 2001), it was predicted that participants with a South-Asian background would have a more global focus than participants with a Western background. However, the only significant difference between the two samples was that participants in India were overall $165 \mathrm{~ms}$ slower than participants in the UK. Although numerically participants in India showed a stronger global advantage effect than participants in the UK (63 vs. 23 $\mathrm{ms}$, respectively), statistically the interaction did not reach significance $(\mathrm{p}=.29)$. Thus, cultural background neither modulated the overall global advantage nor the occurrence of a sadness-specific global advantage.

A possible reason for not finding a sadness-specific effect in Experiment 1 might be the recurring switches every $20 \mathrm{~s}$ between sad and neutral pictures, which might have flattened the emotional effect of the pictures in subsequent trials. In order to test this hypothesis, we carried out a second experiment, using film sequences for a deeper and more enduring emotion induction, and using a between-subject variation with two groups to avoid emotional flattening. One group was presented with a sad video (underlaid with sad music), whereas the other group was presented with a neutral video.

\section{Experiment 2}

Experiment 2 used the same task and the same letter stimuli as in Experiment 1, with the only difference being the method of emotion induction: Instead of pictures, we used emotional film stimuli (see Rottenberg, Ray, \& Gross et al., 2007, for a discussion). The study again included two samples, one from the UK (Experiment 2a) and one from India (Experiment 2b).

\section{Method}

Participants We aimed to recruit 40 participants from the University of Warwick research participant pool and 40 participants from the University of Allahabad participant pool. Each participant gave informed consent and the study was approved by the Ethics Committees of University of Warwick and University of Allahabad.
Apparatus, stimuli, design and procedure Apparatus, task, design, and procedure were the same as in Experiment 1. The main difference was the induction of sadness. Instead of presenting a picture before each trial, participants were presented with a video accompanied by background music at the beginning of the experiment. Videos and music were chosen to elicit either a sad or a neutral emotional state. In the sad condition a collection of six film sequences was taken from advertisements on driving safety. The sequences typically consisted of scenes involving car accidents and the overall video lasted 4:05 min. In the neutral condition the video consisted of scenes of cars driving without any accidents (3:57 min). Music was played during each video through headphones to enhance the emotional experience of the video. In the sad condition the song "My Immortal" composed by "Evanescence" was played, and in the control condition the song "VCR" composed by the band "The XX" was played. This means that mood was now varied between and not within participants. Another difference to Experiment 1 was that participants completed the DES twice, once at the beginning (i.e., right after the video but before the Navon task), and once at the end of the experiment.

Design The independent variables were the within-subject factor Target Type (global, local) and the between-subject factors Video Condition (sad, neutral) and Sample (UK, India). The dependent variables were RT and error rate. There was a total of 32 experimental trials, with half the participants running in the neutral video and the other half in the sad video condition.

Analysis Mean percentage errors and mean RTs (excluding errors and RT outliers) were calculated for each participant and variable combination. The three-way mixed-design ANOVA had the within-subject factor Target Type (local, global) and the between-subject factors Video Condition (sad, neutral) and Sample (UK, India).

\section{Results}

In total 47 participants signed up in the UK and 40 participants in India to take part in the experiment. Seven participants from the UK sample were not included in the analysis because of their origin (China 2; Korea 2; Singapore 2; Philippines 1). Five participants from the India sample had to be excluded because they wrongly responded only to the identity of the global letter (sad video condition 4; neutral video condition 1). Thus, the following results are based on 40 participants from the UK and 35 participants from India.

Navon letter task Mean RTs (excluding 2.7\% errors and $0.5 \%$ outliers) are presented in Fig. 5. The three-way ANOVA revealed only a significant main effect for Target Type, $\mathrm{F}(1,71)$ $=5.92, \mathrm{p}=.018$ : RTs were overall $68 \mathrm{~ms}$ faster for global than 

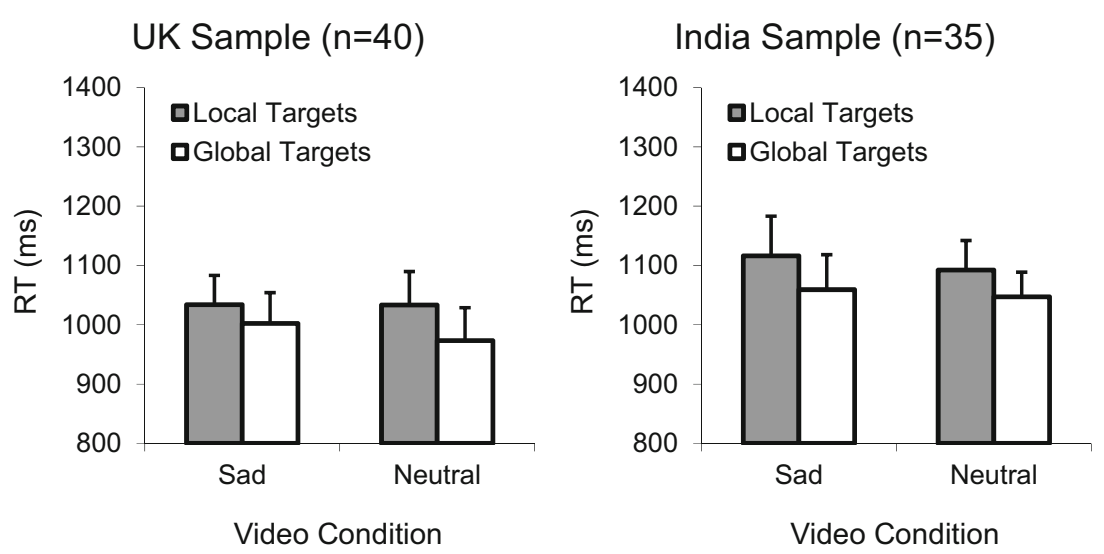

Fig. 5 Mean reaction times (RTs) and SEM in Experiment 2 as a function of video condition (sad, neutral) and target type (local, global), in separate graphs for the UK sample and the India sample

for local targets. Note that none of the effects involving Video Condition were significant (Target Type $\times$ Video Condition, $\mathrm{F}(1,71)=0.04 \mathrm{p}=.89 ; 3$-way interaction, $\mathrm{F}(1,71)=0.25, \mathrm{p}=$ $.62)$. The absence of the Target Type $\times$ Video Condition interaction was substantiated in the Bayesian ANOVA, which showed strong evidence in favor of the null hypothesis $\left(\mathrm{BF}_{\text {Inclusion }}=0.05\right)$. The overall global advantage did differ between samples, $\mathrm{F}(1,71)=0.08, \mathrm{p}=.77$.

The overall error rate was $2.7 \%$ and the error pattern (see Fig. 6) was similar to Experiment 1, with a unique global advantage following sad videos, Target Type $\times$ Video Condition interaction, $\mathrm{F}(1,71)=5.18, \mathrm{p}=.026$. Note that the same interaction was also significant when errors were $\log$ transformed. However, the ANOVA with the inverse efficiency scores (see Fig. 6) found the same two-way interaction to be not significant, $\mathrm{F}(1,71)=.44, \mathrm{p}=51$, and neither was the three-way interaction, $\mathrm{F}(1,71)=0.59, \mathrm{p}=.48$.

Differential emotions scale (DES) The emotional ratings collected at the beginning (after the video) and at the end of the experiment are shown in Table 3 (columns 2-5). Ratings were overall lower in the UK sample than in the Indian sample (1.9 vs. 2.4, respectively), with the exception of sadness ( 2.7 vs. 2.7, respectively). The sadness ratings were analyzed with a 3-way ANOVA with the between-subject factors Video Condition (sad, neutral) and Sample (UK, India), and the within-subject factor Time (T1, T2). There was a significant effect for Video Condition, $F(1,71)$ $=28.44, \mathrm{p}<.001$, and a significant interaction between Video Condition and Time, $\mathrm{F}(1,71)=33.71, \mathrm{p}<.001$. As can be seen from Table 3, participants watching the sad video rated themselves as sadder than participants watching the neutral video (T1: 3.6 vs. 1.7, respectively); however, this effect did not last until the end of the experiment (T2: 2.7 vs. 2.7, respectively). Table 2 also shows a similar pattern for the other nine emotions (inverted for positive emotions), with differences at $\mathrm{T} 1$ (all $\mathrm{p}<.04$ ), but not at T2 (all $\mathrm{p}>.16$ ). This means that the sad video did not only increase sadness, but to some extent also fear, disgust, anxiety, anger, and it reduced the level of amusement and elatedness. Finally, the emotion ratings (at T1) did not correlate with the global advantage score (all $\mathrm{r}$ between -.06 and .12), except for surprise, $r=.27, p=.018$, which correlated even stronger for participants that watched the sad video, $\mathrm{r}=.38, \mathrm{p}=.023$. This suggests that surprise could have had a broadening effect on attention.
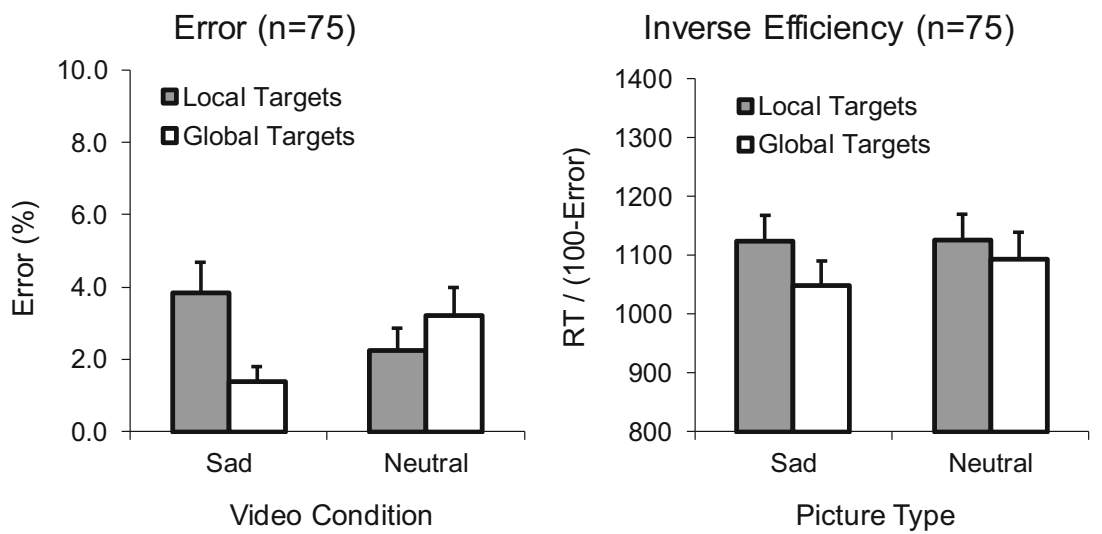

Fig. 6 Mean errors (left graph) and mean inverse efficiency scores (right graph) in Experiment 2, as a function of video condition and target type 


\section{Discussion}

The main purpose of Experiment 2 was to replicate the Gable and Harmon-Jones (2010) study and to use a deeper and more enduring emotion induction. The current findings were much in line with Experiment 1, showing no effect in the RTs, but a sadness-specific global effect in the errors. Note that, although statistically significant, the error effect is numerically rather small and based on only eight trials per factor combination. When we adjusted the RTs for errors by the inverse efficiency calculation, the adjusted pattern of results was once more not markedly different from the original RT pattern. As before, cultural background did not really matter in this experiment. The global advantage was very similar in the India sample compared to the UK sample (51 vs. $46 \mathrm{~ms}$, respectively). This disagrees with the hypothesis that participants would have a more global focus in the Navon task when they had a South-Asian background compared to a Western background (e.g., Boduroglu et al., 2009; Masuda \& Nisbett, 2001).

Another purpose of Experiment 2 was to use a deeper and more enduring emotion induction than in Experiment 1. In terms of emotion induction, the sad video had a marked effect on the sadness rating in the DES, even though this effect did wear off at the end of the experiment. One could argue that maybe the sadness-induced global advantage did not occur because the effect of the sad video was too weak and too short-lived. Even though this possibility cannot be ruled out with certainty, we would like to point out that videos have been shown to be more effective than pictures in terms of inducing and enhancing a prolonged emotional state (see Rottenberg et al., 2007, Schaefer, Nils, Sanchez, \& Philippot, 2010). The other nine ratings in the DES collected at the beginning also indicated that the sad video did not solely affect sadness, but also fear, disgust, anxiety, and anger. This is in line with the literature, showing that it is difficult - if not impossible - to induce sadness in the experimental laboratory without other negative emotions (Izard, Bartlett, \& Marshall 1972; Schwartz \& Weinberger, 1980). Of course, one could argue that the current emotion induction simply induced a general negatively valenced mood. But given the effect was clearly strongest in the sadness rating, we would argue that it primarily induced sadness, with some weaker co-variation of other negative emotions.

\section{General discussion}

This paper reports two experiments aiming to replicate Gable and Harmon-Jones (2010) finding that sadness leads to attentional broadening. Both experiments failed to find such an effect in the RTs, but both found a small effect in the error rates. A broader analysis of the RT data showed that the absence of a sadness effect is neither accounted for by the use of anomalous pictures (with regards to the IAPS picture ratings), nor by the inclusion of anomalous participants (with regards to the DES ratings). Moreover, these findings did not depend on the cultural background of the participants. The observed sadness-induced global advantage in the error rates provides partial support for Gable and Harmon-Jones' (2010) motivational intensity account. It also illustrates the importance of reporting and analyzing error rates in studies using RTs as its primary measure.

One possible interpretation of the error effect is that sadness does not only broaden attention, but it also hinders the continuous tuning of the speed-accuracy threshold, which determines when to abort the stimulus processing in order to give a best-guess response. The absence of error feedback on a trial-by-trial basis might have added to these difficulties, but again this would not explain the difference between this and Gable and Harmon-Jones' (2010) study, as they both used the same feedback regime.

In an attempt to combine RTs and errors into a single performance measure, we calculated inverse efficiency score. In both experiments, these overall performance scores showed no significant interactions that would support a sadnessspecific global advantage. Besides, it has also been shown that inverse efficiency scores can be problematic in terms of linearity and increased variance, and that they can overestimate an effect (e.g., Bruyer \& Brysbaert, 2011). An alternative solution would be to analyze only the RTs from participants that made no errors. In total across both experiments, there were 76 faultless participants. They showed a slightly larger global advantage with sad than with neutral emotion ( 33 vs. $8 \mathrm{~ms}$, respectively), but this difference was statistically not significant, $\mathrm{t}(75)=0.81, \mathrm{p}=.42$.

Experiment 1 was designed to replicate Gable and Harmon-Jones' (2010) experiment as closely as possible and there is hardly any difference between the two studies that could account for the different outcomes. The most obvious difference is that Gable and Harmon-Jones' sample was from North America whereas our samples were from the UK and India. Maybe North American participants are different in terms of felt emotions than UK or Indian participants. Even though this possibility cannot be ruled out entirely, the fact that there were no differences in the picture ratings between the two studies (e.g., the pictures were rated as similarly sad) does not support this explanation.

Another possibility is that cultural differences might play a role in the deployment and setting of attention. For example, Nisbett and Miyamoto (2005) showed that Western cultures tend to be more analytic than Eastern cultures and this is thought to have its roots in the way the two cultures view the world. For example, Western cultures emphasize the importance of being goal-orientated and the need to be analytical to achieve this goal, whereas Eastern cultures prioritize harmony and collective action, which is a much more holistic 
approach. However, such differences do not provide a solution to the discrepancy between our and Gable and Harmon-Jones' (2010) study, because our UK and their North-American samples are both part of the Western culture. Of course, we cannot rule out that other differences of this type exist between the North-American and the UK culture, but in our study, the cultural comparison did no support the idea that culture matters for the sadness-induced global advantage effect. In other words, in our study two very different cultures did not show a sadness-induced global advantage effect, making cultural difference an unlikely reason for the lack of the effect. This problem can only be addressed empirically, with a further replication of Gable and Harmon-Jones' (2010) using a North American sample.

Experiment 2 used a different methodology (film and music) to induce a deeper and more enduring sadness, but again, this did not have much of a broadening effect of attention. There are a number of differences in the methodological procedure that one could draw on in order to explain this absence of an effect (e.g., absence of picture stimuli, emotion induction through films, between-subject design). However, at this point we refrain from further speculations, as we believe future explorations will foremost focus on Experiment 1, which represents a much closer replication of Gable and Harmon-Jones study.

Gable and Harmon-Jones' $(2008,2010)$ motivational intensity account predicts that sadness is associated with a failure to obtain goals, which should therefore broaden attention to aid the consideration of alternative options. Another prediction of their account is that emotions high in motivational intensity precede goal-directed behavior, which should narrow attention to help with the achievement of goals. For example, Gable and Harmon-Jones (2008) showed that desire, which is high in motivational intensity, had a narrowing effect on attention, whereas humor, which is low in motivational intensity, had a broadening effect on attention. Domachowska et al. (2016) carried out an independent replication of Gable and Harmon-Jones's (2008). The authors were able to replicate the original study showing that positive pictures that were approach-motivated narrowed attention compared to neutral pictures.

Does sadness have a broadening effect on attention? The current study cannot give the ultimate answer to this question, mainly because at this point we cannot provide a satisfactory explanation for the discrepancies between the two studies. However, when looking at the broader picture, then our study is well in line with a number of studies on that same topic listed in Table 1, which found no effect - or a narrowing effect of sadness on attention. Such discrepancies across studies on an empirical level points to the need for more independent replication studies (see LeBel, Vanpaemel, McCarthy, Earp, \& Elson, 2017), and on a theoretical level indicates that the impact of emotion induction techniques on subsequent attentional processes need to be better understood.

\section{References}

Basso, M. R., Schefft, B. K., Ris, M. D., \& Dember, W. N. (1996). Mood and global-local visual processing. Journal of the International Neuropsychological Society, 2(3), 249-255.

Bellaera, L., \& von Muhlenen, A. (2017). The effect of induced sadness and moderate depression on attention networks. Cognition and Emotion, 31(6), 1140-1152.

Bellaera, L., von Mühlenen, A., \& Watson, D. G. (2014). When being narrow minded is a good thing: Locally biased people show stronger contextual cueing. The Quarterly Journal of Experimental Psychology, 67(6), 1242-1248.

Bodenhausen, G. V., Sheppard, L. A., \& Kramer, G. P. (1994). Negative affect and social judgment: The differential impact of anger and sadness. European Journal of Social Psychology, 24(1), 45-62.

Boduroglu, A., Shah, P., and Nisbett, R. E. (2009). Cultural differences in allocation of attention in visual information processing. Journal of Cross Cultural Psychology, 40, 349-360.

Bradley, M. M., \& Lang, P. J. (1994). Measuring emotion: The selfassessment manikin and the semantic differential. Journal of Behavior Therapy and Experimental Psychiatry, 25(1), 49-59.

Bradley, M. M. \& Lang, P. J. (2007). Emotion and motivation. In J. T. Cacioppo, L. G. Tassinary \& G. Bernston (Eds.), Handbook of psychophysiology (3rd, pp. 581-607). New York: Cambridge University Press.

Bruyer, R., \& Brysbaert, M. (2011). Combining speed and accuracy in cognitive psychology: Is the inverse efficiency score (IES) a better dependent variable than the mean reaction time (RT) and the percentage of errors (PE)? Psychologica Belgica, 51(1), 5-13.

Chepenik, L. G., Cornew, L. A., \& Farah, M. J. (2007). The influence of sad mood on cognition. Emotion, 7(4), 802-811.

Clore, G. L., \& Palmer, J. (2009). Affective guidance of intelligent agents: How emotion controls cognition. Cognitive Systems Research, 10(1), 21-30.

Clore, G. L., Wyer, R. S., Dienes, B., Gasper, K., Gohm, C., \& Isbell, L. (2001). Affective feelings as feedback: Some cognitive consequences. Theories of mood and cognition: A user's guidebook (pp. 27-62). Mahwah, NJ: Lawrence Erlbaum Associates, Inc.

Cohen, J. (1988). Statistical power analysis for the behavioral sciences (2nd). Hillsdale: Lawrence Erlbaum Associates.

Derryberry, D., \& Tucker, D. M. (1994). Motivating the focus of attention. In P. M. Niedenthal \& S. Kitayama (Eds.), The heart's eye: Emotional influences in perception and attention (pp. 167-196). San Diego: Academic Press.

Domachowska, I., Heitmann, C., Deutsch, R., Goschke, T., Scherbaum, S., \& Bolte, A. (2016). Approach-motivated positive affect reduces breadth of attention: Registered replication report of Gable and Harmon-Jones (2008). Journal of Experimental Social Psychology, $67,50-56$.

Duncan, S., \& Barrett, L. F. (2007). Affect is a form of cognition: A neurobiological analysis. Cognition and Emotion, 21(6), 1184-1211.

Easterbrook, J. A. (1959). The effect of emotion on cue utilization and the organization of behavior. Psychological Review, 66(3), 183-201.

Faul, F., Erdfelder, E., Lang, A.-G., \& Buchner, A. (2007). G*Power 3: A flexible statistical power analysis program for the social, behavioral, and biomedical sciences. Behavior Research Methods, 39, 175-191.

Fenske, M. J., \& Eastwood, J. D. (2003). Modulation of focused attention by faces expressing emotion: evidence from flanker tasks. Emotion, 3(4), 327-343.

Finucane, A. M., Whiteman, M. C., \& Power, M. J. (2010). The effect of happiness and sadness on alerting, orienting, and executive attention. Journal of Attention Disorders, 13(6), 629-639.

Fredrickson, B. L., \& Branigan, C. (2005). Positive emotions broaden the scope of attention and thought-action repertoires. Cognition \& Emotion, 19(3), 313-332. 
Gable, P. A., \& Harmon-Jones, E. (2008). Approach-motivated positive affect reduces breadth of attention. Psychological Science, 19(5), 476-482.

Gable, P. A., \& Harmon-Jones, E. (2010). The blues broaden, but the nasty narrows: Attentional consequences of negative affects low and high in motivational intensity. Psychological Science, 21(2), 211-215.

Gable, P. A., \& Harmon-Jones, E. (2013). Does arousal per se account for the influence of appetitive stimuli on attentional scope and the late positive potential? Psychophysiology, 50(4), 344-350.

Gable, P. A., \& Harmon-Jones, E. (2016). Assessing the Motivational Dimensional Model of emotion-cognition interaction: Comment on Domachowska, Heitmann, Deutsch, et al., (2016). Journal of Experimental Social Psychology, 67, 57-59.

Gasper, K., \& Clore, G. L. (2002). Attending to the big picture: Mood and global versus local processing of visual information. Psychological Science, 13(1), 34-40.

Huntsinger, J. R. (2012). Does positive affect broaden and negative affect narrow attentional scope? A new answer to an old question. Journal of Experimental Psychology: General, 141(4), 595-600.

Huntsinger, J. R., Clore, G. L., \& Bar-Anan, Y. (2010). Mood and globallocal focus: Priming a local focus reverses the link between mood and global-local processing. Emotion, 10(5), 722-726.

Izard, C. E., Bartlett, E. S., \& Marshall, A. G. (1972). Patterns of emotions. New York: Academic Press.

Izard, C. E., Dougherty, F. E., Bloxom, B. M., \& Kotsch, N. E. (1974). The Differential Emotions Scale: A method of measuring the meaning of subjective experience of discrete emotions. Nashville: Vanderbilt University, Department of Psychology.

JASP Team (2016). JASP (Version 0.8.0.0) [Computer software].

Kimchi, R., \& Palmer, S. E. (1982). Form and texture in hierarchically constructed patterns. Journal of Experimental Psychology: Human Perception and Performance, 8(4), 521-535.

Kringelbach, M. \& Phillips, H. (2014). Emotion: Pleasure and Pain in the Brain. Oxford University Press: Oxford

Lang, P. J., Bradley, M. M., \& Cuthbert, B. N. (2005). International Affective Picture System (IAPS): Affective ratings of pictures and instruction manual. Technical Report A-6. Gainesville, FL: The Center for Research in Psychophysiology, University of Florida.

LeBel, E. P., Vanpaemel, W., McCarthy, R. J., Earp, B. D., \& Elson, M. (2017). A Unified Framework to Quantify the Trustworthiness of Empirical Research. Retrieved from psyarxiv.com/uwmr8

Masuda T., \& Nisbett R. E. (2001) Attending holistically vs. analytically: Comparing the context sensitivity of Japanese and Americans. Journal of Personality and Social Psychology, 81(5), 922-934.

Melcher, T., Obst, K., Mann, A., Paulus, C., \& Gruber, O. (2012). Antagonistic modulatory influences of negative affect on cognitive control: Reduced and enhanced interference resolution capability after the induction of fear and sadness. Acta Psychologica, 139(3), 507-514.

Mottus, R., Allik, J., Realo, A., Rossier, J., Zecca, G., Ah-Kion, J., ... Johnson, W. (2012). The Effect of Response Style on Self-Reported Conscientiousness Across 20 Countries. Personality and Social Psychology Bulletin, 38(11), 1423-1436.

Navon, D. (1977). Forest before trees: The precedence of global features in visual perception. Cognitive Psychology, 9(3), 353-383.

Nisbett, R.E., \& Miyamoto, Y. (2005). The influence of culture: Holistic versus analytic perception. Trends in Cognitive Sciences, 9, 467473.

Philippot, P. (1993). Inducing and assessing differentiated emotionfeeling states in the laboratory. Cognition \& Emotion, 7(2), 171-193.

Rottenberg, J., Ray, R. D., \& Gross, J. (2007) Emotion elicitation using films. In J. A. Coan \& J. J. Allen (Eds.), Handbook of emotion elicitation and assessment (pp. 9-28), New York: Oxford University Press.

Rowe, G., Hirsh, J. B., \& Anderson, A. K. (2007). Positive affect increases the breadth of attentional selection. Proceedings of the National Academy of Sciences, 104(1), 383-388.

Schaefer, A., Nils, F., Sanchez, X., \& Philippot, P. (2010). Assessing the effectiveness of a large database of emotion-eliciting films: A new tool for emotion researchers. Cognition \& Emotion, 24(7), 11531172

Schwartz, G. E., \& Weinberger, D. A. (1980). Patterns of emotional responses to affective situations: Relations among happiness, sadness, anger, fear, depression, and anxiety. Motivation and Emotion, 4, 175-191.

Srinivasan, N., \& Gupta, R. (2010). Emotion-attention interactions in recognition memory for distractor faces. Emotion, 10(2), 207-215.

Srinivasan, N., \& Hanif, A. (2010). Global-happy and local-sad: Perceptual processing affects emotion identification. Cognition and Emotion, 24(6), 1062-1069.

Tan, H. K., Jones, G. V., \& Watson, D. G. (2009). Encouraging the perceptual underdog: Positive affective priming of nonpreferred local-global processes. Emotion, 9(2), 238-247.

Townsend, J. T., \& Ashby, F. G. (1983). The stochastic modeling of elementary psychological processes. New York: Cambridge University Press.

Uddenberg, S., \& Shim, W. M. (2015). Seeing the world through targettinted glasses: Positive mood broadens perceptual tuning. Emotion, 15(3), 319-328.

Wagenmakers, E.-J. (2007). A practical solution to the pervasive problems of p values. Psychonomic Bulletin \& Review, 14(5), 779-804.

Wetzels, R., Matzke, D., Lee, M. D., Rouder J.N., Iverson, G. J., \& Wagenmakers, E.-J. (2011). Statistical Evidence in Experimental Psychology: An Empirical Comparison Using 855 t Tests. Perspectives on Psychological Science, 6(3), 291-298. 